\title{
Cocaine Increases Actin Cycling: Effects in the Reinstatement Model of Drug Seeking
}

\author{
Shigenobu Toda, Hao-Wei Shen, Jamie Peters, Stephanie Cagle, and Peter W. Kalivas \\ Department of Neurosciences, Medical University of South Carolina, Charleston, South Carolina 29464
}

\begin{abstract}
Addiction to cocaine is associated with persistent changes in synaptic function. The cycling of actin between polymerized [ $\mathrm{F}$ (for filamentous)] and depolymerized forms contributes to synaptic plasticity, and acute and withdrawal from repeated cocaine administration produced reversible and enduring elevations, respectively, in F-actin in the nucleus accumbens. Increased F-actin after 3 weeks withdrawal from repeated cocaine resulted from changes in the content or phosphorylation state of actin binding proteins (ABPs) that cosediment with F-actin. The profile of altered APBs was consistent with filopodia formation, including increased mammalian Enabled, phosphorylated (p)-cortactin, and p-vasodilator-stimulated phosphoprotein, and increased actin depolymerization [e.g., reduced LIM (Lin11/Isl-1/Mec3)-kinase and p-cofilin]. In contrast to repeated cocaine, the increase in F-actin after acute cocaine administration resulted from reduced depolymerization and actin cycling. The potential involvement of chronic cocaine-induced increases in actin cycling in cocaine addiction was examined using the reinstatement of cocaine seeking in rats previously trained to self-administer cocaine by inhibiting actin polymerization with intra-accumbens latrunculin $\mathrm{A}$ or by accelerating actin depolymerization with a LIM-kinase inhibitor. Disrupting actin cycling via either mechanism augmented cocaine-induced reinstatement of drug seeking but did not affect the locomotor response to acute cocaine administration. Thus, withdrawal from repeated cocaine induces a restructuring of actin-ABP complexes, which increases actin cycling and may modulate cocaine-induced reinstatement of drug seeking.
\end{abstract}

Key words: actin; cocaine; cofilin; reinstatement; LIM-kinase; accumbens

\section{Introduction}

Actin assembly and disassembly (actin cycling) regulates dendritic spine morphology and protein insertion into the postsynaptic density (PSD) (Rao and Craig, 2000; Ehlers, 2002; Krause et al., 2003; McGee and Bredt, 2003; Matus, 2005; Wang et al., 2005). Actin cycling is controlled by various actin binding proteins (ABPs) that maintain equilibrium between monomeric [G (for globular)] and filamentous [F (for filamentous)] actin (dos Remedios et al., 2003). The polymerization of F-actin occurs by ABP binding to a site of F-actin assembly, termed a barbed end, which regulates the stability and elongation of F-actin into filopodia and lamellipodia (dos Remedios et al., 2003; Mejillano et al., 2004). Similarly, the disassembly of F-actin is promoted by ABPs (Ono, 2003). Both the polymerization and depolymerization of F-actin are modulated, in part, by phosphorylation of the ABPs, thereby linking actin cycling to neurotransmission and associated kinase signaling cascades (McGee and Bredt, 2003; Carlisle and Kennedy, 2005).

Excitatory synapses in the brain are primarily on dendritic spines, and molecular adaptations within dendritic spines mediate stimulus-induced neuroplasticity in excitatory transmission, such as long-term potentiation (Nimchinsky et al., 2002; Lisman,

Received Aug. 8, 2005; revised Nov. 27, 2005; accepted Dec. 18, 2005.

This work was supported in part by United States Public Health Service Grants DA-12513 and DA-05369.

Correspondence should be addressed to Dr. Peter W. Kalivas, Department of Neurosciences, Medical University of

South Carolina, 173 Ashley Avenue, BSB 403, Charleston, SC 29464. E-mail: kalivasp@musc.edu.

DOl:10.1523/JNEUROSCI.4132-05.2006

Copyright $\odot 2006$ Society for Neuroscience $\quad$ 0270-6474/06/261579-09\$15.00/0
2003). Changes in cell signaling and dendritic spine morphology are thought to contribute to behavioral adaptations induced by motivationally relevant life events, including the emergence of psychopathologies such as addiction (Robinson and Kolb, 1997; McGee and Bredt, 2003; Blanpied and Ehlers, 2004; Kalivas et al., 2005). Thus, animal models of psychostimulant-induced behavioral plasticity and addiction are associated with enduring adaptations in dendritic proteins such as Homer and PSD-95 (Swanson et al., 2001; Yao et al., 2004), particularly within nuclei regulating behavioral responding to reward, such as the prefrontal cortex and nucleus accumbens (Everitt and Wolf, 2002; Kalivas et al., 2005).

The critical role played by actin cycling in dendritic plasticity and function indicates that the involvement of dendritic spines and scaffolding proteins in animal models of addiction could be associated with alterations in actin. The regulation of actin cycling by ABPs and $\mathrm{ABP}$ involvement in dendritic spine morphology has been characterized in cell culture experiments and using mice with deletions of genes encoding ABPs (Meng et al., 2002; dos Remedios et al., 2003; Rabenstein et al., 2005). Although in vivo imaging studies have confirmed actin-based motility of dendritic spines (Majewska and Sur, 2003; Matus, 2005), direct in vivo evidence supporting a role for rearrangement of actin in enduring behavioral adaptations is sparse in general (Fischer et al., 2004) and absent in the field of addiction. The present experiments were designed to determine whether acute and repeated cocaine administration rearranges actin, as evidenced by changes in the level and/or phosphorylation state 
of F-actin and the ABPs regulating actin cycling. Moreover, inhibition of actin polymerization by microinjecting latrunculin A into the nucleus accumbens or promoting actin depolymerization and branching by inhibiting LIM (Lin11/Isl-1/Mec3)-kinase (LIMK) was used to determine whether actin rearrangement can affect cocaine-induced drug seeking after withdrawal from cocaine self-administration.

\section{Materials and Methods}

Animal housing. Male Sprague Dawley rats weighing 250-275 g (Harlan Sprague Dawley, Indianapolis, IN) were housed in groups of two, with food and water available ad libitum. A $12 \mathrm{~h}$ light/dark cycle was used with lights on at 7:00 A.M. All saline $(\mathrm{Sa})$ and cocaine $(\mathrm{Coc})$ injections were performed during light cycle. All experiments were conducted according to specifications of National Institutes of Health Guide for the Care and Use of Laboratory Animals.

Reagents and antibodies. Cocaine, amphetamine, and morphine were provided by the $\mathrm{Na}$ tional Institute on Drug Abuse (Bethesda, MD). The antibodies used were as follows: anti-actin (1:100; Santa Cruz Biotechnology, Santa Cruz, CA), anti-cofilin (cof) (1:500; BD Transduction Laboratories, San Diego, CA), antiphosphorylated (p)-cofilin (1:1000; Chemicon, Temecula, CA), anti-LIMK-1 (1:250; BD Transduction Laboratories), anti-profilin (1:1000; Alexis, San Diego, CA), anti-fascin (1: 2000; BD Transduction Laboratories), antiMena (1:250; BD Transduction Laboratories), anti-actin-related protein 3 (Arp3) (1:500; BD Transduction Laboratories), anti-p-adducin (1:1000; Upstate Biotechnology, Lake Placid, NY), anti-p-vasodilator-stimulated phosphoprotein (VASP) (Ser 157) (1:250; Cell Signaling Technology, Beverly, MA), anti-p-cortactin (Tyr 421) (1: 1000; Stressgen, Victoria, British Columbia, Canada), anti-PSD-95 (1: 1000; Sigma, St. Louis, MO), anti-glutamate receptor 1 (GluR1) (1:1000; Upstate Biotechnology), anti-Homerlb/c (1:200; Santa Cruz Biotechnology), anti-NMDA receptor 1 (NMDAR-1) (1:1000; Upstate Biotechnology), and anti-NMDAR-2A (1:1000; Upstate Biotechnology). Tat peptides were synthesized and purified at the Biotechnology Resource Laboratory (Medical University of South Carolina, Charleston, SC). Tatcof (MASGVAVSDGVIKVFNGRRRRRRRRRRR) contained the phosphorylation site of cofilin fused with a Tat-like poly-arginine membrane permeability sequence and was previously shown to inhibit LIM-kinase in neuronal culture experiments (Yang et al., 1998; Aizawa et al., 2001). Tat-scrambled fusion peptide (sc) (NFVKIVGDSVAVGSAMGRRRRRRRRRRR) contained the reverse sequence of the cofilin phosphorylation site and was used as a control.

Drug administration and tissue preparation. All rats were acclimatized to the housing facility for 1 week before beginning daily drug injections (for treatment protocol, see Fig. 1a). Rats were treated with either daily saline or cocaine in their home cages (for $7 \mathrm{~d} ; 15 \mathrm{mg} / \mathrm{kg}$, i.p., on the first and last day; $30 \mathrm{mg} / \mathrm{kg}$, i.p., on the intervening $5 \mathrm{~d}$ ). At 3 weeks after the last daily injection, the animals were decapitated $45 \mathrm{~min}$ after an acute injection of saline or cocaine $(30 \mathrm{mg} / \mathrm{kg}$, i.p.) for immunoblotting. Some groups were microinjected into the nucleus accumbens with latrunculin A, $1 \%$ DMSO, or Tat-fusion peptides (see below) and decapitated 45 or 135 min later, and tissue was prepared for immunoblotting. Alternatively, the effect of latrunculin A or Tat-fusion proteins on cocaineinduced locomotor behavior and reinstatement was examined. In one experiment, rats were treated with daily amphetamine $(1 \mathrm{mg} / \mathrm{kg}$, i.p., on the first and last day; $2.5 \mathrm{mg} / \mathrm{kg}$, i.p., on the intervening $5 \mathrm{~d}$ ) or morphine
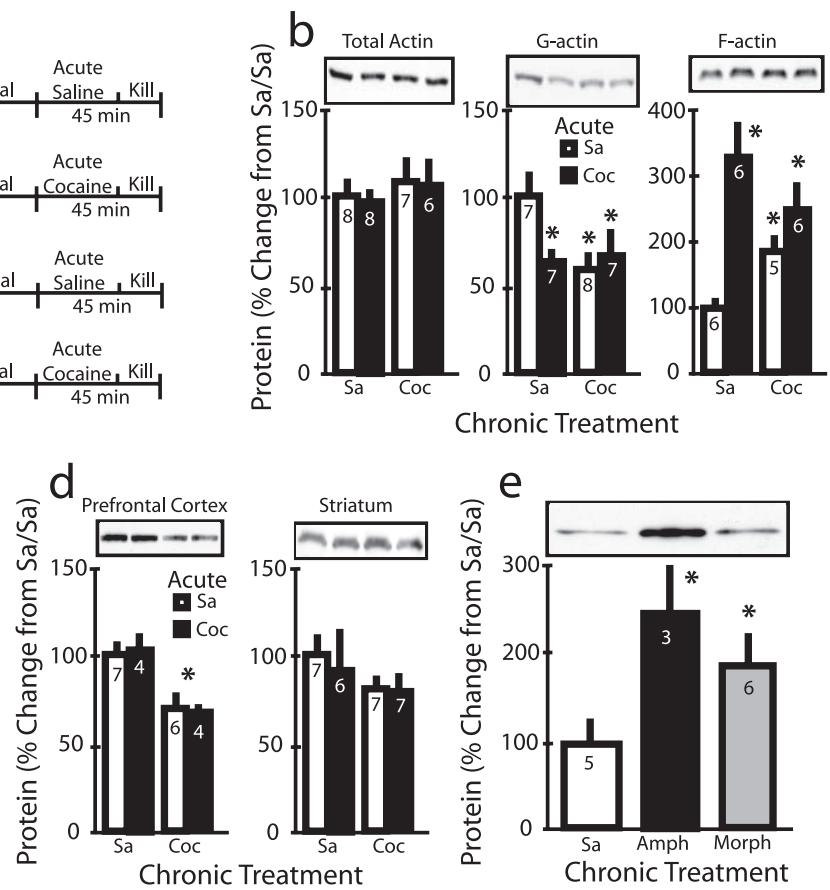

Figure 1. Acute and repeated cocaine elevate F-actin in the accumbens. $\boldsymbol{a}$, Cocaine treatment regimens: Sa/Sa, repeated saline/acute saline; $\mathrm{Sa} / \mathrm{Coc}$, repeated saline/acute cocaine; $\mathrm{Coc} / \mathrm{Sa}$, repeated cocaine/acute saline; $\mathrm{Coc} / \mathrm{Coc}$, repeated cocaine/ acute cocaine. $\boldsymbol{b}$, Acute and repeated cocaine elevate F-actin and reduce $\mathrm{G}$-actin without affecting total actin at $45 \mathrm{~min}$ after , ANOVA: $\boldsymbol{b}$, F-actin, interaction, $F_{(1,20)}=6.19, p=0.022 ; \mathrm{G}$-actin, interaction, $F_{(1,25)}=5.31, p=0.030 ; \boldsymbol{d}$ prefrontal cortex, chronic, $F_{(1,17)}=7.90, p=0.012 ; \boldsymbol{e}, F_{(2,10)}=5.78, p=0.022$. ${ }^{*} p<0.05$ compared with Sa/Sa $(\boldsymbol{b})$ or

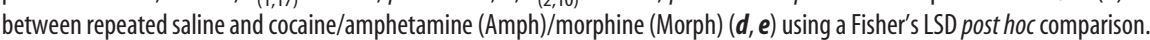

( $3 \mathrm{mg} / \mathrm{kg}$, i.p., on the first and last day; $10 \mathrm{mg} / \mathrm{kg}$, i.p., on the intervening $5 \mathrm{~d})$ and then killed 3 weeks later.

Subcellular fractionation and immunoblotting. Subcellular fractionation was performed on dissected brain tissue as described in detail previously (Toda et al., 2003). All buffers contained $1 \mathrm{~nm}$ microcystin LR (Sigma), $2 \mathrm{~mm}$ sodium pyrophosphate, $1 \mathrm{~mm} \mathrm{Na}_{3} \mathrm{VO}_{4}, 5 \mathrm{~mm} \mathrm{NaF}, 10 \mathrm{~mm}$ sodium pyrophosphate, $20 \mathrm{~mm} \beta$-glycerophosphatase, $0.1 \mathrm{~mm}$ ammonium molybdate, $1 \mathrm{~mm}$ para-nitrophenylphosphatase, $1 \mathrm{~mm}$ benzamide, and Complete Mini including EDTA (1 tablet/10 ml; Roche Products, Indianapolis, IN) to prevent degradation and dephosphorylation of proteins. F-actin in synaptosomal membrane fraction was separated from G-actin as described previously (Fukazawa et al., 2003). Briefly, synaptosomal membrane fraction was resuspended in a buffer A (1\% Triton $\mathrm{X}-100,20 \mathrm{~mm}$ HEPES-NaOH, and $100 \mathrm{~mm} \mathrm{NaCl}, \mathrm{pH}$ 7.2) and incubated at $4^{\circ} \mathrm{C}$ for $1 \mathrm{~h}$ with gentle rotation. After a $20 \mathrm{~min}$ centrifugation $\left(10,000 \times g, 4^{\circ} \mathrm{C}\right)$, the pellet was resuspended in buffer B $(15 \mathrm{~mm}$ HEPES$\mathrm{NaOH}, 0.15 \mathrm{~mm} \mathrm{NaCl}, 1 \%$ SDS, 10 mm EDTA, and 1 mm DTT, pH 7.5). After $1 \mathrm{~h}$ of incubation at $4^{\circ} \mathrm{C}$ and an additional 20 min centrifugation $\left(10,000 \times g, 4^{\circ} \mathrm{C}\right)$, the second supernatant was recovered as the F-actinenriched fraction. Because the first supernatant contains little actin, the $\mathrm{G}$-actin fraction was extracted from total protein minus the nuclear fraction as buffer A-soluble supernatant. Immunoblotting was performed as described previously (Toda et al., 2003). Briefly, 2-50 $\mu \mathrm{g}$ of protein was used, and immunoreactivity of actin, LIMK-1, cofilin, p-cofilin, and profilin were detected from total protein. The immunoreactivities of PSD95, GluR1, NMDAR-1, NMDAR-2A, Arp3, fascin, Homer1b/c, Mena, $\mathrm{p}$-VASP, p-cortactin, and p-adducin were detected from the F-actin fraction.

Surgery and microinjection. Surgery followed 1 week of acclimation to daily handling. Rats were stereotaxically implanted with guide cannulas (26 gauge) under ketamine $(87.5 \mathrm{mg} / \mathrm{kg}$ Ketaset; Fort Dodge Animal Health, Fort Dodge, IA) and xylazine (5 mg/kg Rompum; Bayer, Shaw- 
nee Mission, KS) anesthesia in the nucleus accumbens at $+0.6 \mathrm{~mm}$ anterior, $\pm 1.8 \mathrm{~mm}$ lateral at an angle of $6^{\circ}$ from vertical, and $-4.0 \mathrm{~mm}$ ventral from bregma (Paxinos and Watson, 1986). Some animals were also implanted with intravenous jugular catheters for subsequent cocaine self-administration, as described previously (Bowers et al., 2004). Latrunculin A (Invitrogen, Carlsbad, CA) was dissolved in DMSO [1\% solution in artificial CSF (aCSF) containing (in mM) 5 D-glucose, $2.7 \mathrm{KCl}$, $140 \mathrm{NaCl}, 1.2 \mathrm{MgCl}_{2}$, and $1.4 \mathrm{CaCl}_{2}$ ]. Tat peptides were diluted in aCSF plus $1 \%$ glycerol. One week after surgery, bilateral infusion cannulas (33 gauge) were inserted, extending $1 \mathrm{~mm}$ beyond the tip of the guide cannulas. Infusions were in a volume of $1 \mu$ l over $2 \mathrm{~min}$, and an additional 2 min was allowed for diffusion before the infusion cannulas were removed. Microinjections were made $5 \mathrm{~min}$ (latrunculin) or $30 \mathrm{~min}$ (Tatfusion peptide) before systemic injections of cocaine or saline. For both the biochemical and behavioral studies, $0.5 \mu \mathrm{g}$ of latrunculin A or 1.0 nmol of Tat peptide was microinjected. These doses were based in part on the in vivo literature (Bowers et al., 2004; Fischer et al., 2004) and preliminary in vivo studies showing effects of different doses of microinjected latrunculin and Tat-cof on F-actin in Coc/Sa subjects and p-cofilin in $\mathrm{Sa} / \mathrm{Coc}$ subjects, respectively.

Cocaine-induced locomotion. Locomotor activity was monitored in a photocell apparatus (Omnitech, Columbus, $\mathrm{OH}$ ). Motor activity was quantified as total distance traveled (determined by consecutive breaking of adjacent photobeams) and stereotypy (consecutive breaking of the same photobeam). For these sessions, animals were allowed to habituate to the activity chambers for $60 \mathrm{~min}$ before microinjection of latrunculin A or Tat peptide, which was followed by cocaine $(15 \mathrm{mg} / \mathrm{kg}$, i.p.), and behavior was recorded for $120 \mathrm{~min}$. Behavior was assessed over $5 \mathrm{~min}$ intervals. A second behavioral test was performed after a 1 week interval, and subjects were microinjected with the counterbalanced drug or control.

Cocaine self-administration. To facilitate learning self-administration of cocaine, all rats were given a single $15 \mathrm{~h}$ training session in an operant conditioning chamber fitted with two retractable levers (ENV-008; Med Associates, East Fairfield, VT), and each lever press on the correct lever was reinforced by delivery of a single $45 \mathrm{mg}$ food pellet. Lever presses on the inactive lever had no programmed consequences. Surgery was performed $2 \mathrm{~d}$ after food training, and intravenous cocaine selfadministration training began $7 \mathrm{~d}$ after surgery. Cocaine selfadministration training was conducted during $2 \mathrm{~h}$ sessions during the rat's dark cycle. Rats were trained to press a lever using an fixed ratio 1 schedule of cocaine reinforcement $(0.3 \mathrm{mg} / \mathrm{kg}$ cocaine hydrochloride in the latrunculin experiment or $0.6 \mathrm{mg} / \mathrm{kg}$ in the Tat-fusion peptide experiment, administered in $0.05 \mathrm{ml}$ over $4 \mathrm{~s}$ ) with a $20 \mathrm{~s}$ timeout after each infusion. Correct lever presses resulted in cocaine infusion and illumination of a stimulus light over the lever. The light remained on for $20 \mathrm{~s}$, during which time correct lever presses were counted but resulted in no additional cocaine infusion. After the $20 \mathrm{~s}$ timeout, the light turned off. Responses on the inactive lever had no programmed consequences but were recorded. Data collection and cocaine delivery were controlled using Schedule Manager for Windows software version 2.09 (Med Associates). Daily training sessions lasted $2 \mathrm{~h}$ or until a subject earned 200 cocaine infusions, whichever came first. Animals remained in selfadministration training until they obtained at least 20 infusions per day on a minimum of 10 consecutive self-administration days and exhibited $<20 \%$ variability in active lever responding on 3 consecutive days.

Extinction and reinstatement testing. Extinction training commenced the day after a rat achieved acquisition criteria (see above). The house light was illuminated throughout each session, and responses were recorded on both levers but had no programmed consequences. Rats received extinction training on a minimum of 7 consecutive days or until the responding on the active lever fell to $<20 \%$ of the active lever pressing during maintenance for 3 consecutive days. The day after achieving this criterion, subjects were returned to the operant chambers for reinstatement testing. Subjects were microinjected with Tat-cofilin peptide or latrunculin A before reinstatement testing. Cocaine $(10 \mathrm{mg} / \mathrm{kg}$, i.p.) was administered 5 min (latrunculin A or 1\% DMSO) or 30 min (Tat-fusion peptide) after microinjection. Animals were placed in the selfadministration chambers for a $2 \mathrm{~h}$ evaluation of lever pressing. During reinstatement testing, active lever presses were counted but resulted in no reinforcement. For the latrunculin experiment, animals were tested only once for cocaine-induced reinstatement, and separate animals were treated with either latrunculin or DMSO control injections. For the Tatfusion peptide experiment, subjects were tested twice for reinstatement to cocaine, and each animal received Tat-cof and Tat-sc in random order, separated by more than three extinction trials.

Histology and statistical analysis. When microinjection cannulas were implanted and animals were not used for immunoblotting, the brains were perfused with Formalin (10\%) via intracardiac infusion, sliced (100 $\mu \mathrm{m}$ thick), and section stained with cresyl violet. Location of microinjection cannula tips were identified using light microscopy and a stereotaxic atlas (Paxinos and Watson, 1986). The cannulas were predominately in the core subcompartment of the accumbens, and animals without both cannula in the accumbens (shell or core) were removed before data analysis.

A two-tailed Student's $t$ test (paired or unpaired) or a one- or two-way ANOVA (with or without repeated measures over time) followed by a Fisher's least significant difference test was used to evaluate all neurochemical and behavioral data. $F, t$, and probability values are reported only for significant $(p<0.05)$ effects.

\section{Results \\ Acute cocaine and withdrawal from repeated cocaine increase F-actin}

After 3 weeks of withdrawal from 1 week of daily saline or cocaine administration $(15 \mathrm{mg} / \mathrm{kg}$ on days 1 and $7 ; 30 \mathrm{mg} / \mathrm{kg}$ on days $2-6)$, rats were injected with saline or cocaine $(30 \mathrm{mg} / \mathrm{kg}$, i.p.) and killed $45 \mathrm{~min}$ later (Fig. 1a). The levels of total actin were not altered in any cocaine treatment group (Fig. 1b). Actin cycles between G-actin and F-actin (Pollard and Borisy, 2003) and both acute cocaine $(\mathrm{Sa} / \mathrm{Coc})$ and withdrawal from repeated cocaine $(\mathrm{Coc} / \mathrm{Sa})$ increased the level of F-actin and reduced the level of $\mathrm{G}$-actin in the accumbens compared with control subjects ( $\mathrm{Sa}$ / Sa) (Fig. 1b). However, acute cocaine administered to animals withdrawn from repeated cocaine ( $\mathrm{Coc} / \mathrm{Coc}$ ) did not further alter the levels of F- or G-actin beyond the changes produced by chronic cocaine (Coc/Sa). The increase in F-actin by acute cocaine in control subjects $(\mathrm{Sa} / \mathrm{Coc})$ was transient, because the levels were not elevated at $120 \mathrm{~min}$ after cocaine administration (Fig. 1c). In contrast to the nucleus accumbens, acute cocaine did not alter the levels of F-actin in either the prefrontal cortex or striatum (Fig. 1d). Whereas withdrawal from repeated cocaine also had no statistically significant impact on the level of F-actin in the striatum, levels in the prefrontal cortex were significantly reduced in both cocaine withdrawal treatment groups (Coc/Sa and $\mathrm{Coc} / \mathrm{Coc})($ Fig. $1 d)$. Akin to cocaine, 3 weeks after withdrawal from 1 week of daily amphetamine $(1 \mathrm{mg} / \mathrm{kg}$, i.p., days 1 and $7 ; 2.5$ $\mathrm{mg} / \mathrm{kg}$, i.p., days 2-6) or morphine (3 mg/kg, i.p., days 1 and 7; 10 $\mathrm{mg} / \mathrm{kg}$, i.p., days 2-6), the levels of F-actin in the accumbens were elevated relative to rats withdrawn from repeated saline (Fig. 1e), indicating that other addictive drugs known to alter dendritic spine morphology also alter actin cycling (Robinson and Kolb, 1997, 1999). However, it is noteworthy that, although withdrawal from amphetamine or cocaine increases, morphine reduces accumbens spine density (Robinson and Kolb, 1997, 1999; Robinson et al., 2001). Similarly, repeated cocaine increases spine density in both the prefrontal cortex and accumbens (Robinson et al., 2001), yet F-actin was elevated in accumbens and lowered in the prefrontal cortex. Thus, alterations in F-actin by addictive drugs may contribute to changes in dendrite morphology but do not appear to determine the direction of enduring morphological changes. 

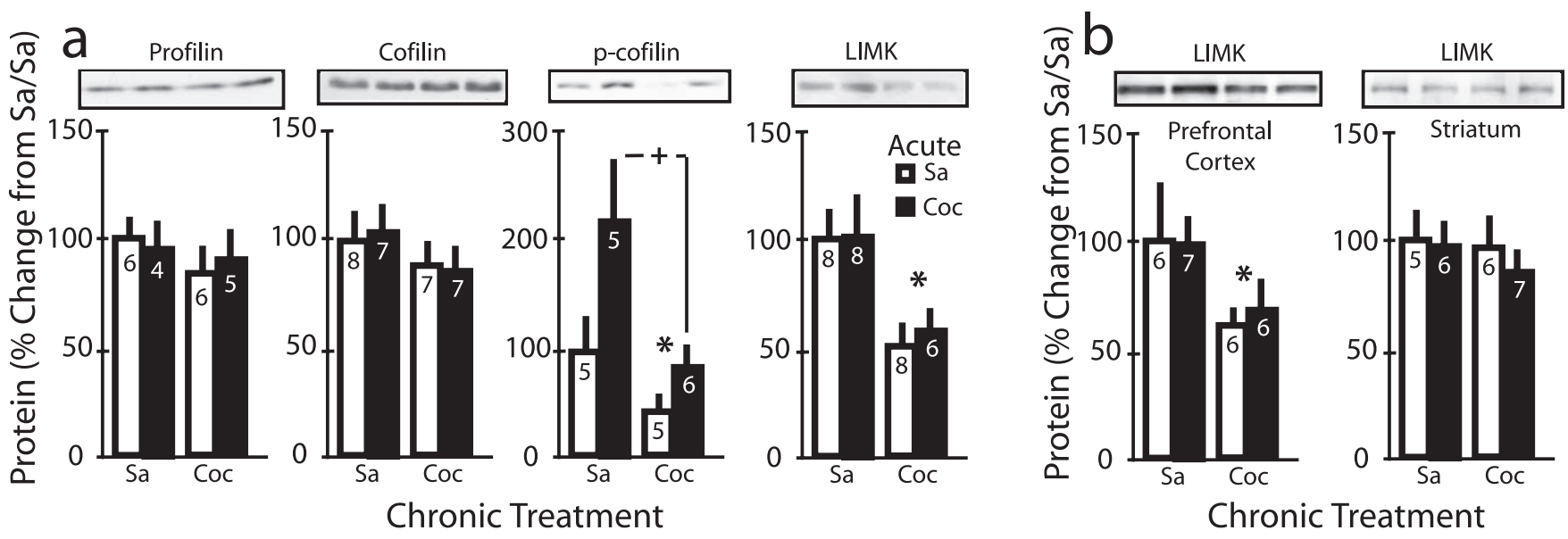

Figure 2. Cocaine alters ABPs that regulate actin depolymerization. For treatment regimens, see Figure 1a. $\boldsymbol{a}$, Effect of cocaine on ABPs in the nucleus accumbens. $\boldsymbol{b}$, Effect of cocaine on LIMK in the prefrontal cortex. Data are shown as mean \pm SEM, and the number of determinations is shown in each bar. Data were evaluated using a two-way ANOVA (acute $\times$ chronic): $p$-cofilin, acute, $F_{(1,17)}=6.87, p=0.018$; chronic, $F_{(1,17)}=8.81, p=0.009 ;$ LIMK, chronic, $F_{(1,26)}=6.21, p=0.019 ;$ prefrontal cortex, chronic, $F_{(1,27)}=4.70, p=0.039$. ${ }^{*} p<0.05$ comparing repeated saline with repeated cocaine. ${ }^{+} p<0.05$ comparing acute saline with acute cocaine.

\section{Acute and chronic cocaine differentially affect ABPs regulating actin depolymerization}

Actin cycling can be regulated by the rate of depolymerization, which is controlled in part by the ABPs cofilin and profilin (Ono, 2003). The total tissue content of neither cofilin nor profilin was altered in the accumbens by acute or withdrawal from repeated cocaine (Fig. $2 a$ ). The ability of cofilin to bind and depolymerize F-actin is regulated by LIMK phosphorylation of cofilin, which markedly reduces its binding affinity for F-actin (Ono, 2003). In contrast to total cofilin content, p-cofilin was markedly and differentially regulated by acute and withdrawal from repeated cocaine. Thus, acute cocaine administration ( $\mathrm{Sa} / \mathrm{Coc}$ and $\mathrm{Coc} / \mathrm{Coc}$ ) elevated p-cofilin compared with acute saline $(\mathrm{Sa} / \mathrm{Sa}$ and $\mathrm{Coc} /$ $\mathrm{Sa}$ ), whereas withdrawal from repeated cocaine (Coc/Sa and Coc/ $\mathrm{Coc}$ ) reduced $\mathrm{p}$-cofilin compared with repeated saline ( $\mathrm{Sa} / \mathrm{Sa}$ and $\mathrm{Sa} / \mathrm{Coc}$ ) (Fig. 2a). Akin to F-actin, the rise in p-cofilin by acute cocaine $(\mathrm{Sa} / \mathrm{Coc})$ was reversible, and, $120 \mathrm{~min}$ after cocaine injection, the levels had returned to normal ( $\mathrm{Sa} / \mathrm{Sa}, 100 \pm 16.0 \%$, $\left.n=7 ; \mathrm{Sa} / \mathrm{Coc}, 67.8 \pm 14.3 \%, n=7 ; t_{(12)}=1.50 ; p=0.160\right)$. The reduced level of $\mathrm{p}$-cofilin after withdrawal from repeated cocaine likely resulted from a marked reduction in LIMK-1 in the accumbens of cocaine-withdrawn subjects (Fig. 2a). The level of LIMK-1 was also examined in the prefrontal cortex and striatum. Although there was no alteration in LIMK-1 in the striatum or prefrontal cortex after acute cocaine, the level of LIMK-1 was reduced in the prefrontal cortex by withdrawal from repeated cocaine but not altered in the striatum (Fig. $2 b$ ). These data dissociate the effects of cocaine withdrawal on F-actin from LIMK-1, because F-actin was elevated in the accumbens and reduced in the prefrontal cortex, but LIMK-1 was lowered in both regions by repeated cocaine (compare Figs. $1 b, d, 2 b$ ).

Increased F-actin subfractionating with ABPs associated with filopodia-like protein complexes

An increase in F-actin can polymerize in relatively linear structures termed filopodia or as multi-branched complexes termed lamellipodia, and the formation of filopodia versus lamellipodia is controlled by the association of distinct ABPs with F-actin (dos Remedios et al., 2003; Mejillano et al., 2004). The formation of lamellipodia occurs at sites on F-actin that have been nucleated by cofilin and subsequently bound by the Arp2/3 protein complex (May, 2001). Arp3 levels were not altered in the accumbens
F-actin subfraction of any cocaine treatment group, indicating that lamellipodia-like protein complexes were not formed (Fig. 3). Two ABPs indicative of filopodia formation are fascin and the Enabled (Ena)/VASP protein complex. There was a trend for fascin to be elevated in all cocaine treatment groups, whereas the level of the Ena/VASP protein Mena was significantly increased by withdrawal from repeated cocaine (Fig. 3). Ena/VASP binding to F-actin has two known consequences. (1) Ena/VASP inhibits Arp2/3 binding to F-actin, thereby decreasing F-actin branching and lamellipodia formation (Krause et al., 2003). (2) Capping proteins bind to the barbed end of F-actin to prevent polymerization, and the phosphorylation of VASP by protein kinase A or $\mathrm{G}$ inhibits the binding of capping proteins (Krause et al., 2003; Svitkina et al., 2003). The level of p-VASP was increased in accumbens after withdrawal from repeated cocaine administration (Fig. 3). Together, the elevation in Mena and phosphorylation of VASP after repeated cocaine are consistent with the preferential polymerization of F-actin as filopodia-like versus lamellipodialike protein complexes.

Cortactin promotes the nucleation of F-actin, thereby facilitating binding of the Arp2/3 complex and lamellipodia formation (Lua and Low, 2005). However, phosphorylation by Src kinase reduces the binding affinity of cortactin for F-actin and Arp2/3, thereby limiting the formation of lamellipodia (Lua and Low, 2005). Consistent with an elevation in ABPs that promote filopodia-like protein complexes, an antibody selective for Src kinase phosphorylation at Tyr 421 of cortactin detected an increase in p-cortactin after withdrawal from repeated cocaine (Coc/Sa and Coc/Coc) (Fig. 3).

Adducin is a capping protein, and phosphorylation by protein kinase $\mathrm{C}$ decreases the binding affinity of adducin for the barbed end of F-actin, thereby permitting actin polymerization (Matsuoka et al., 2000). Although withdrawal from repeated cocaine (Coc/Sa and $\mathrm{Coc} / \mathrm{Coc}$ ) did not alter adducin phosphorylation, the increase in F-actin by acute cocaine ( $\mathrm{Sa} / \mathrm{Coc}$ and $\mathrm{Coc} / \mathrm{Coc}$ ) was associated with increased levels of p-adducin (Fig. 3).

Withdrawal from repeated cocaine increases actin cycling The data shown in Figure 3 indicated that withdrawal from repeated cocaine increased actin cycling by regulating the binding of ABPs to F-actin. To disrupt actin cycling and inhibit actin polymerization, latrunculin $\mathrm{A}(0.5 \mu \mathrm{g} / \mu \mathrm{l})$ was microinjected into 

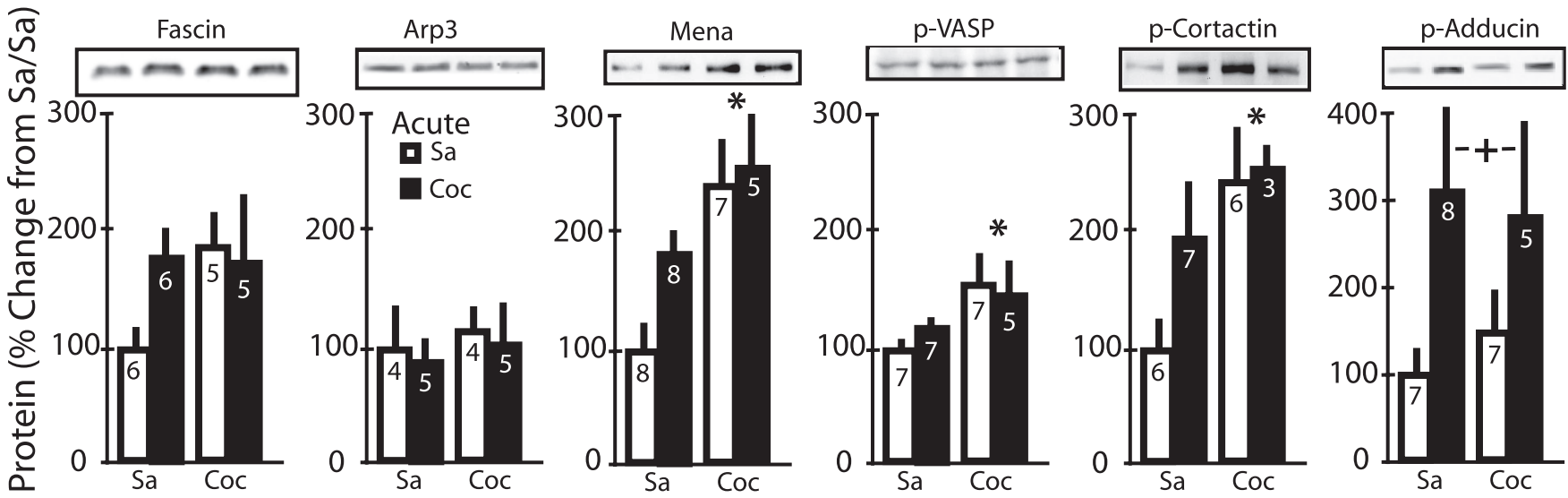

\section{Chronic Treatment}

Figure 3. Cocaine alters ABPs that regulate actin polymerization. For treatment regimens, see Figure $1 a$. Data are shown as mean $\pm S E M$, and the number of determinations is shown in each bar. The data were evaluated using a two-way ANOVA: Mena, chronic, $F_{(1,24)}=8.09, p=0.009 ; p$-VASP, chronic, $F_{(1,22)}=4.87, p=0.038 ; p$-cortactin, chronic, $F_{(1,18)}=5.24, p=0.039 ; p$-adducin, acute, $F_{(1,23)}=4.36, p=0.048 .{ }^{*} p<0.05$ comparing repeated cocaine (Coc/Sa, $\left.\mathrm{Coc} / \mathrm{Coc}\right)$ with repeated saline (Sa/Sa, $\left.\mathrm{Coc} / \mathrm{Sa}\right) .{ }^{+} p<0.05$ comparing acute saline $(\mathrm{Sa} / \mathrm{Sa}$, $\mathrm{Coc} / \mathrm{Sa})$ with acute cocaine $(\mathrm{Sa} / \mathrm{Coc}, \mathrm{Coc} / \mathrm{Coc})$.
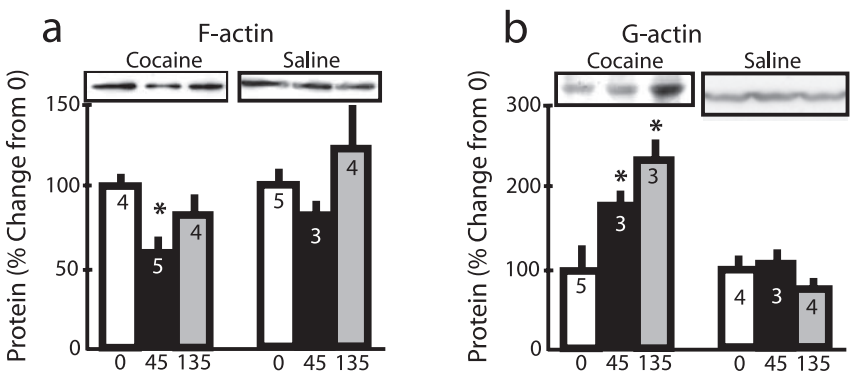

Figure 4. Inhibition of actin cycling alters F-actin and G-actin. Three weeks after discontinuing repeated saline or cocaine, rats were injected into the nucleus accumbens with latrunculin $A$ $(0.5 \mu \mathrm{g})$ or DMSO (10\%) vehicle and killed immediately or 45 or 135 min later, and the levels of $\mathrm{F}$-actin and $\mathrm{G}$-actin were examined. Data are shown as mean $\pm \mathrm{SEM}$, and the number of determinations is shown in each bar. Data were evaluated using a one-way ANOVA: cocaine F-actin, $F_{(2,10)}=6.23, p=0.020$; cocaine G-actin, $F_{(2,8)}=4.48, p=0.050 .{ }^{*} p<0.05$ compared with time 0 .

the accumbens of rats withdrawn from chronic cocaine (Coc/Sa) or saline $(\mathrm{Sa} / \mathrm{Sa})$, and the animals were killed immediately or 45 or 135 min later. Latrunculin binds directly to G-actin, thereby preventing actin polymerization (Morton et al., 2000). Conversely, latrunculin A elevated the level of G-actin in the accumbens (Fig. 4b). However, in contrast with F-actin, the levels of G-actin did not moderate at 135 min after microinjection of latrunculin A, perhaps indicative of the fact that latrunculin A increases actin synthesis, which would be reflected first as depolymerized (G) actin (Morton et al., 2000). In contrast to withdrawal from repeated cocaine, no significant effect on F- or G-actin content was measured in animals pretreated with repeated saline, and latrunculin A elicited a reversible reduction in F-actin in cocaine-withdrawn rats (Fig. 4). These data indicated that actin cycling was lower in control relative to cocainewithdrawn subjects and is consistent with in vivo observations that, in adult animals, dendritic spines are relatively stable compared with spines in developing animals (Matus, 2005). The rise in F-actin elicited by acute cocaine administration in repeated saline-pretreated subjects $(\mathrm{Sa} / \mathrm{Coc})$ was not inhibited by latrunculin A in the accumbens (DMSO, $100 \pm 24.8 \%, n=3$; latrunculin A, $124.4 \pm 23.6 \%, n=3)$.
Table 1. Lack of effect by acute and repeated cocaine on various PSD proteins cosedimenting with F-actin

\begin{tabular}{lcccc}
\hline Treatment & PSD-95 & GluR1 & NMDA-R1 & Homer1b/c \\
\hline Sa/Sa & $100 \pm 13(7)$ & $100 \pm 16(7)$ & $100 \pm 11(6)$ & $100 \pm 9(6)$ \\
Sa/Coc & $95 \pm 15(7)$ & $104 \pm 19(7)$ & $154 \pm 21(7)$ & $105 \pm 23(6)$ \\
Coc/Sa & $89 \pm 13(4)$ & $113 \pm 35(5)$ & $150 \pm 35(5)$ & $109 \pm 22(5)$ \\
Coc/Coc & $99 \pm 14(5)$ & $99 \pm 33(5)$ & $131 \pm 24(5)$ & $80 \pm 2(3)$ \\
\hline
\end{tabular}

\section{Cocaine did not alter the level of PSD proteins in the}

\section{F-actin fraction}

Because actin cycling is thought to affect the insertion of proteins into the PSD (Rao and Craig, 2000; McGee and Bredt, 2003), a number of PSD proteins associated with glutamate transmission were measured in the F-actin subfraction of the accumbens after 3 weeks of withdrawal from repeated cocaine or saline treatments. Given the increase in F-actin induced by acute ( $\mathrm{Sa} / \mathrm{Coc}$ ) and withdrawal from repeated cocaine $(\mathrm{Coc} / \mathrm{Sa})$, it was surprising that the content of the PSD proteins measured, including PSD95, NMDAR-1, GluR1, and Homer1b/c, was unaltered by any cocaine treatment (Table 1).

\section{Do changes in actin cycling contribute to} cocaine-induced behaviors?

To determine whether the changes produced in F-actin cycling and ABPs by acute and chronic cocaine are of behavioral significance, two pharmacological manipulations were made in the accumbens, and effects on cocaine-induced locomotor activity and the reinstatement of cocaine seeking were quantified. As outline above, latrunculin A was microinjected into the accumbens to inhibit actin polymerization (Morton et al., 2000). In addition, the role of depolymerization was examined by inhibiting LIMK with a peptide antagonist consisting of the phosphorylation domain of cofilin fused to the membrane permeability domain of human immunodeficiency virus-1 (HIV)-Tat (Yang et al., 1998; Aizawa et al., 2001; Bowers et al., 2004). Compared with a scrambled fusion peptide control injection (Tat-sc), the LIMK antagonist (Tat-cof) significantly reduced the rise in p-cofilin in the accumbens elicited by acute cocaine administration (Fig. $5 a$ ). In contrast, inhibiting LIMK did not alter p-cofilin in saline-treated 

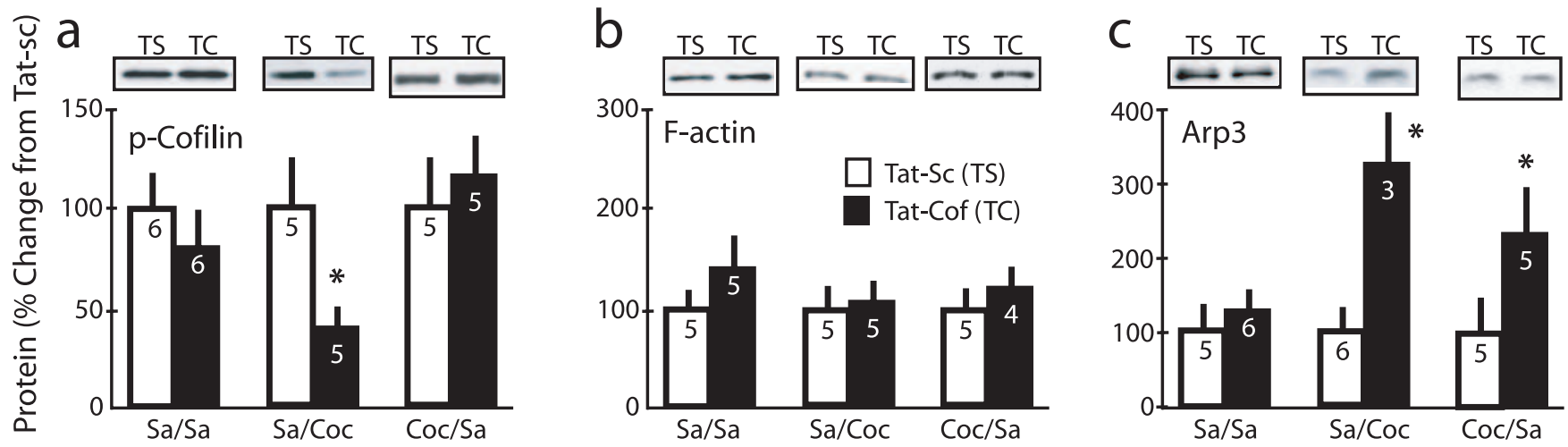

Figure 5. Inhibition of LIMK prevents acute cocaine-induced increase in p-cofilin. Three weeks after discontinuing repeated saline or cocaine (see Fig. 1a), rats were injected into the nucleus accumbens with a peptide antagonist of LIMK fused to the membrane permeability domain of HIV-Tat ( $1 \mathrm{nmol}$; Tat-cof) or a scrambled fusion peptide control ( $1 \mathrm{nmol}$; Tat-sc), injected 30 min later with cocaine $(30 \mathrm{mg} / \mathrm{kg}$, i.p.) or saline, and killed $45 \mathrm{~min}$ later, and F-actin-enriched subfraction of accumbens was examined for various proteins. Data are shown as mean $\pm \mathrm{SEM}$, and the number of determinations is shown in each bar and were statistically evaluated using a two-tailed Student's $t$ test: Sa/Coc, p-cofilin, $t_{(8)}=2.91, p=0.020 ; S_{1} / C_{0 c}$, Arp3, $t_{(7)}=2.34, p=0.051 ; C_{0} / S a$, Arp3, $t_{(8)}=2.63, p=0.030 .^{*} p<0.05$ comparing Tat-cof with Tat-sc.

animals (Fig. 5a), supporting the conclusion from the latrunculin A experiment (Fig. 4a) that basal actin cycling is less in control compared with cocaine-treated subjects. Also, the inhibition by Tat-cof did not affect the levels of p-cofilin in cocaine-withdrawn animals (Fig. $5 a$ ), presumably because basal LIMK levels were already markedly reduced in this group (Fig. $2 a$ ). Inhibition of LIMK did not alter F-actin content in chronic saline ( $\mathrm{Sa} / \mathrm{Sa})$, acute cocaine $(\mathrm{Sa} / \mathrm{Coc})$, or chronic cocaine $(\mathrm{Coc} / \mathrm{Sa})$ subjects (Fig. $5 b$ ). The reduction in $\mathrm{p}$-cofilin by Tat-cof would be predicted to increase cofilin binding to F-actin and thereby reduce F-actin content by promoting depolymerization (Ono, 2003). This may not have occurred in part because the binding of cofilin to F-actin nucleates the filament creating potential branch points for the binding of Arp2/3 and cortactin to promote lamellipodia formation (Meng et al., 2002; Ono, 2003; Ghosh et al., 2004). Thus, it is possible that the lack of effect by LIMK inhibition on F-actin levels may result from an equilibration between increased depolymerization and simultaneous lamellipodia-like formation. Supporting this possibility, Arp3 levels were increased in the acute cocaine and withdrawal from repeated cocaine treatment groups ( $\mathrm{Sa} / \mathrm{Coc}$ and $\mathrm{Coc} / \mathrm{Sa}$ ) after LIMK inhibition (Fig. $5 c$ ).

In the reinstatement model, rats were trained to selfadminister cocaine, and lever pressing was subsequently extinguished by removing cocaine reinforcement. Rats were then administered cocaine $(10 \mathrm{mg} / \mathrm{kg}$, i.p.) to reinstate active lever pressing. Before cocaine, rats were pretreated with latrunculin A, Tat-cof, or corresponding control microinjection into the accumbens. Both Tat-cof $(1.0 \mathrm{nmol})$ and latrunculin A $(0.5 \mu \mathrm{g})$ significantly augmented cocaine-induced active lever pressing compared with the respective control treatments (Fig. 6a). Neither extinction lever pressing the day before the cocaine test nor inactive lever pressing during the cocaine test was different between the drug treatment groups (Fig. 6a).

Locomotor behavior was measured in a photocell apparatus and quantified as distance traveled (estimate of locomotion) and stereotypy counts. Pretreatment with either latrunculin A (0.5 $\mu \mathrm{g})$ or Tat-cof $(1 \mathrm{nmol})$ did not reduce the increase in distance traveled (Fig. $6 b$ ) or stereotypy (data not shown) elicited by cocaine injection (15 mg/kg, i.p.).

\section{Discussion}

This study demonstrates that withdrawal from repeated cocaine produced an enduring increase in F-actin and actin cycling in the
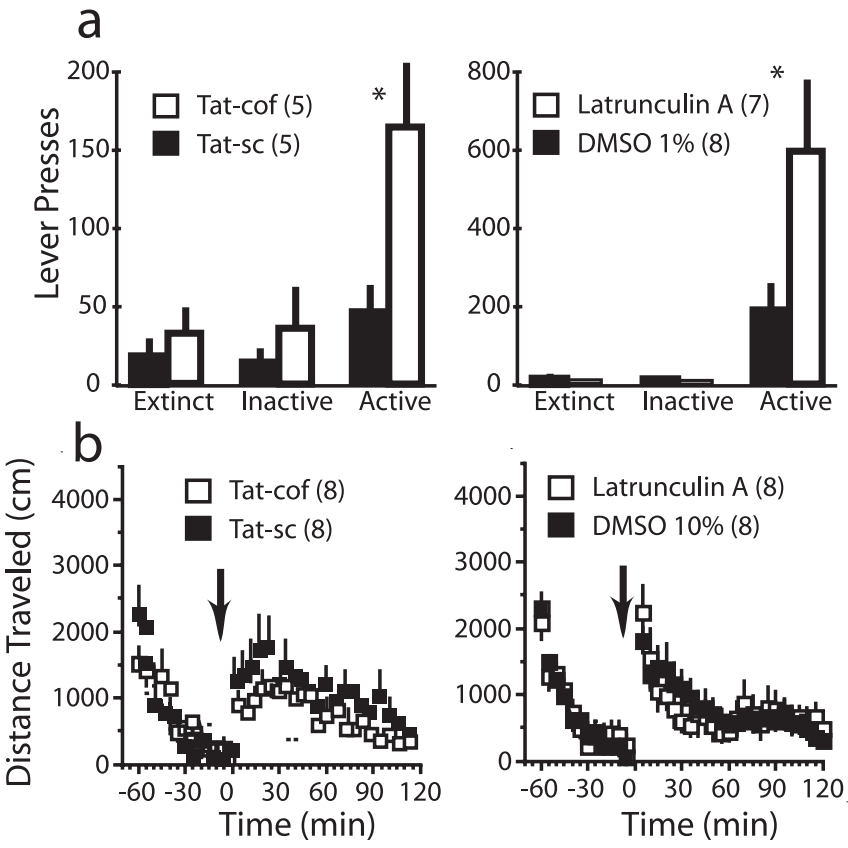

Figure 6. Inhibition of actin cycling potentiates cocaine-induced reinstatement of drug seeking. $\boldsymbol{a}$, Rats were extinguished from cocaine self-administration and given cocaine (10 $\mathrm{mg} / \mathrm{kg}$, i.p.) to reinstate lever pressing. Thirty minutes before cocaine reinstatement, animals were microinjected with Tat-cof $(1 \mathrm{nmol})$ or Tat-sc $(1 \mathrm{nmol})$ or, $5 \mathrm{~min}$ before cocaine, were microinjected with latrunculin $(0.5 \mu \mathrm{g})$ or DMSO (1\%) vehicle. Data are shown as mean \pm SEM active lever presses during the extinction trial the day before reinstatement (Extinct) and inactive and active lever presses during reinstatement. The number of determinations is shown in parentheses. For the latrunculin experiment, each animal received only one reinstatement trial, and the data were evaluated with an unpaired Student's $t$ test (latrunculin active lever, $t_{(13)}=$ 2.24; $p=0.043)$. For the Tat-peptide experiment, each animal received two reinstatement trials, one each with Tat-cof or Tat-sc in random order, and the data were analyzed using a paired Student's $t$ test (Tat-cof active lever, $t_{(5)}=2.53 ; p=0.052$ ). $\boldsymbol{b}$, Separate rats were pretreated with intra-accumbens drug as in the reinstatement study, and the acute locomotor response was examined in a photocell apparatus. Data are shown as mean \pm SEM distance traveled, and cocaine ( $15 \mathrm{mg} / \mathrm{kg}$, i.p.) was administered at the time indicated by the arrow. ${ }^{*} p<0.05$ comparing drug with control.

nucleus accumbens, and the polymerization of actin was associated with alterations in ABPs that associate with filopodia-like protein complexes (summarized in Table 2). Acute cocaine administration also increased F-actin, but the rise was transient and appears to result primarily from decreased depolymerization via 
Table 2. Summary of changes in actin and ABPs produced by acute and withdrawal from repeated cocaine

\begin{tabular}{|c|c|c|c|}
\hline \multicolumn{2}{|c|}{ Acute cocaine } & \multicolumn{2}{|c|}{ Repeated cocaine } \\
\hline Increase & Decrease & Increase & Decrease \\
\hline $\begin{array}{l}\text { F-actin } \\
\text { p-cofilin } \\
\text { p-adducin }\end{array}$ & G-actin & $\begin{array}{l}\text { F-actin } \\
\text { Mena }^{b, c} \\
\text { p-VASP } \\
\text { p-cortactin } \\
b, c\end{array}$ & $\begin{array}{l}\text { G-actin } \\
\text { p-cofilin } \\
\text { LIMK-1 }^{a}\end{array}$ \\
\hline
\end{tabular}

${ }^{a}$ Promotes depolymerization.

${ }^{b}$ Promotes polymerization.

'ABPs associated with filopodia.

inactivation (increased phosphorylation) of cofilin. Alterations in F-actin contribute to the remodeling of excitatory synaptic architecture (Rao and Craig, 2000; McGee and Bredt, 2003; Carlisle and Kennedy, 2005; Matus, 2005; Wang et al., 2005), and the enduring changes in F-actin elicited by withdrawal from repeated cocaine may contribute to the dendritic dysmorphisms (Robinson et al., 2001) and alterations in postsynaptic glutamate transmission in the accumbens (Sutton et al., 2003; Boudreau and Wolf, 2005; Kalivas et al., 2005) that have been postulated to underlie cocaine addiction. Accordingly, changing actin cycling by inhibiting actin polymerization with intra-accumbens latrunculin A or increasing depolymerization by LIMK inhibition facilitated cocaine-induced reinstatement of drug seeking.

\section{Adaptations in actin cycling induced by acute cocaine versus withdrawal from repeated cocaine}

Based on associated changes in ABPs, the increase in F-actin elicited by acute versus withdrawal from repeated cocaine relies on different mechanisms. Whereas withdrawal from repeated cocaine simultaneously increased both the depolymerization and polymerization of actin, indicative of increased actin cycling (dos Remedios et al., 2003; Gungabissoon and Bamburg, 2003), acute cocaine was associated primarily with changes consistent with reduced depolymerization and actin cycling (for a summary model, see supplemental Fig. 1, available at www.jneurosci.org as supplemental material). Acute cocaine administration increased the phosphorylation of cofilin by LIMK, which decreases cofilin binding affinity for F-actin. Thus, an elevation in p-cofilin reduces cofilin-induced depolymerization and nucleation of F-actin (Gungabissoon and Bamburg, 2003; Ono, 2003). Slingshot phosphatases and LIMK regulate the reversible phosphorylation of cofilin and exist in a multiprotein complex along with actin (Soosairajah et al., 2005). A role for LIMK in the rise in p-cofilin after acute cocaine is consistent with the fact that inhibiting LIMK by Tat-cof prevented the rise in $\mathrm{p}$-cofilin by acute cocaine, but involvement of slingshot cannot be ruled out. Although the increase in F-actin and reduction in G-actin by acute cocaine arises, in part, from decreased depolymerization (supplemental Fig. 1, available at www.jneurosci.org as supplemental material), polymerization of actin may also contribute because acute cocaine increased adducin phosphorylation, which decreases the binding affinity of this capping protein for the barbed end. However, the fact that inhibiting polymerization with latrunculin A did not decrease F-actin content in acute cocainetreated subjects argues for a primary role of reduced depolymerization (Fig. 4a).

In contrast to acute cocaine, withdrawal from repeated cocaine elicited a marked reduction in LIMK and p-cofilin, which increases cofilin availability and the rate of depolymerization (Ono, 2003), as well as promotes actin nucleation for possible lamellipodia formation (Ghosh et al., 2004). However, the increase in depolymerization by repeated cocaine was not accompanied by increased ABPs associated with lamellipodia-like protein complexes (i.e., no change in Arp3). The apparent lack of recruitment of lamellipodia-associated ABPs cosedimenting with F-actin likely arose from concerted protein changes by withdrawal from repeated cocaine. Notably, the elevation in Ena/ VASP (indicated by elevated Mena) and p-cortactin decreases formation of the Arp2/3-cortactin protein complexes necessary to form lamellipodia at sites of cofilin-induced nucleation (Krause et al., 2003; Lua and Low, 2005). In these circumstances, the increased availability of G-actin by cofilin-mediated depolymerization would be preferentially routed to form filopodia-like protein complexes, as indicated by the rise in Mena. Also, a rise in p-VASP inhibits the binding of capping proteins to the barbed end (Svitkina et al., 2003). Together, the changes in ABPs after withdrawal from repeated cocaine are consistent with a simultaneous elevation in actin polymerization and depolymerization that is indicative of increased actin cycling and changes in dendritic spine function (Meng et al., 2003; Ono, 2003; Pollard and Borisy, 2003; Ghosh et al., 2004) (for modeling of cocaineinduced changes in actin cycling, see supplemental Fig. 1, available at www.jneurosci.org as supplemental material).

\section{Actin cycling and cocaine-induced neuroplasticity}

The cocaine-induced presence of a higher proportion of filopodia-associated ABPs cosedimenting with F-actin in the nucleus accumbens may indicate increased lability of dendritic spines. Supporting this possibility, actin cycling in filopodia is relatively rapid compared with lamellipodia and is associated with the recruitment of proteins into the PSD (Kasai et al., 2003; Matsuzaki et al., 2004). Moreover, the increase in spine density after repeated psychostimulants consists, in part, of an increase in filopodia-like dysmorphisms, such as thin multiheaded spines (Robinson and Kolb, 1997), and deletion of LIMK results in spines with filopodia-like morphology (Meng et al., 2002). Although the present study did not directly assess whether the increase in actin cycling alters glutamate signaling, withdrawal from repeated cocaine induces a number of enduring neuroadaptations in presynaptic and postsynaptic glutamate transmission that may potentially involve actin cycling. The phosphorylation of actin binding proteins, such as synapsin 1, is known to regulate the releasable pool of glutamatergic synaptic vesicles (Rosahl et al., 1995). Repeated psychostimulant administration increases synapsin 1 phosphorylation (Iwata et al., 1996), and the reinstatement of cocaine seeking using a paradigm akin to that in the present study is associated with a large increase in synaptic glutamate release (McFarland et al., 2003). Also, a variety of evidence suggests that enhanced AMPA signaling may occur in the nucleus accumbens of animals withdrawn from repeated cocaine, including increased surface expression of AMPA receptors (Boudreau and Wolf, 2005), increased behavioral responsiveness to AMPA receptor stimulation (Pierce et al., 1996; Suto et al., 2004), and augmented long-term potentiation induced in vivo from the prefrontal cortex (Goto and Grace, 2005) or in vitro via field stimulation (Yao et al., 2004). In contrast, some studies in anesthetized rats or in dissociated cells support a conclusion that repeated cocaine administration renders accumbens neurons relatively less responsive to glutamate (White et al., 1995; Zhang et al., 1998). Importantly, however, it is unknown whether this relatively inhibited state of accumbens neurons persists after a few days of withdrawal. It should also be noted that one study found enhanced long-term depression in the accumbens after repeated 
cocaine (Thomas et al., 2001). However, this phenomenon was observed only in the shell compartment of the accumbens and not in the core compartment that appears to be more critical for cocaine-seeking behaviors (Di Ciano and Everitt, 2001; McFarland and Kalivas, 2001). Although these data together are consistent with withdrawal from repeated cocaine promoting the formation of filopodia-like protein complexes that may contribute to previously reported neuroadaptations in glutamate transmission, it is important to recall that the present study did not directly measure dendritic spine morphology.

\section{Actin and cocaine-induced behavioral plasticity}

Over the past decade, cocaine-induced behavioral plasticity has been strongly associated with adaptations in excitatory transmission in the nucleus accumbens (Wolf, 1998; Kalivas et al., 2005), posing the possibility that altered actin cycling after withdrawal from repeated cocaine may contribute to the expression of these behaviors. Accordingly, intra-accumbens microinjection of either a membrane-permeable peptide LIMK inhibitor or latrunculin A potentiated the capacity of cocaine to reinstate drug seeking. Both of these compounds disrupt the increase in actin cycling and the cosedimenting of F-actin with filopodia-associated ABPs. Inhibition of LIMK increases dephosphorylated cofilin, thereby promoting the formation of lamellipodia-like protein complexes and depolymerization (Ono, 2003), whereas latrunculin A binds to G-actin, prevents the polymerization of actin into filopodialike complexes (Morton et al., 2000). Although a precise mechanism whereby disrupting the increase in actin cycling produced by withdrawal from repeated cocaine promotes the capacity of acute cocaine to reinstate drug seeking was not identified, it appears that it is not by potentiating the acute effect of cocaine because cocaine-induced locomotor activity was not altered by latrunculin A or LIMK inhibition.

\section{Conclusions}

Although actin regulation of dendritic signaling and morphology has been postulated as an important mediator of plasticity associated with neuropsychiatric disorders and neurodegeneration (Kasai et al., 2003; McGee and Bredt, 2003; Blanpied and Ehlers, 2004; Carlisle and Kennedy, 2005), the present study is among the first to indicate that enduring changes in actin cycling may contribute to in vivo behavioral plasticity. Thus, withdrawal from repeated cocaine produced enduring changes in actin cycling by modulating both the level and phosphorylation of ABPs. These adaptations may be a compensatory response to repeated cocaine because disrupting actin cycling potentiated cocaine-induced drug seeking.

\section{References}

Aizawa H, Wakatsuki S, Ishii A, Moriyama K, Sasaki Y, Ohashir K, SekineAizawa Y, Sehara-Fujisawa A, Mizuno K, Goshima Y, Yahara I (2001) Phosphorylation of cofilin by LIM-kinase is necessary for semaphorin 3A-induced growth cone collapse. Nat Neurosci 4:367-373.

Blanpied TA, Ehlers MD (2004) Microanatomy of dendritic spines: emerging principles of synaptic pathology in psychiatric and neurological disease. Biol Psychiatry 55:1121-1127.

Boudreau AC, Wolf ME (2005) Behavioral sensitization to cocaine is associated with increased AMPA receptor surface expression in the nucleus accumbens. J Neurosci 25:9144-9151.

Bowers MS, McFarland K, Lake RW, Peterson YK, Lapish CC, Gregory ML, Lanier SM, Kalivas PW (2004) Activator of G-protein signaling 3: a gatekeeper of cocaine sensitization and drug-seeking. Neuron 42:269-281.

Carlisle HJ, Kennedy MB (2005) Spine architecture and synaptic plasticity. Trends Neurosci 28:182-187.

Di Ciano P, Everitt BJ (2001) Dissociable effects of antagonism of NMDA and AMPA/KA receptors in the nucleus accumbens core and shell on cocaine-seeking behavior. Neuropsychopharmacology 25:341-360.

dos Remedios CG, Chhabra D, Kekic M, Dedova IV, Tsubakihara M, Berry DA, Nosworthy NJ (2003) Actin binding proteins: regulation of cytoskeletal microfilaments. Physiol Rev 83:433-473.

Ehlers MD (2002) Molecular morphogens for dendritic spines. Trends Neurosci 25:64-67.

Everitt BJ, Wolf ME (2002) Psychomotor stimulant addiction: a neural systems perspective. J Neurosci 22:3312-3320.

Fischer A, Sananbenesi F, Schrick C, Spiess J, Radulovic J (2004) Distinct roles of hippocampal de novo protein synthesis and actin rearrangement in extinction of contextual fear. J Neurosci 24:1962-1966.

Fukazawa Y, Saitoh Y, Ozawa F, Ohta Y, Mizuno K, Inokuchi K (2003) Hippocampal LTP is accompanied by enhanced F-actin content within the dendritic spine that is essential for late LTP maintenance in vivo. Neuron 38:447-460.

Ghosh M, Song X, Mouneimne G, Sidani M, Lawrence DS, Condeelis JS (2004) Cofilin promotes actin polymerization and defines the direction of cell motility. Science 304:743-746.

Goto Y, Grace AA (2005) Dopamine-dependent interactions between limbic and prefrontal cortical plasticity in the nucleus accumbens: disruption by cocaine sensitization. Neuron 47:255-266.

Gungabissoon RA, Bamburg JR (2003) Regulation of growth cone actin dynamics by ADF/cofilin. J Histochem Cytochem 51:411-420.

Iwata S, Hewlett GH, Ferrell ST, Czernik AJ, Meiri KF, Gnegy ME (1996) Increased in vivo phosphorylation state of neuromodulin and synapsin I in striatum from rats treated with repeated amphetamine. J Pharmacol Exp Ther 278:1428-1434.

Kalivas PW, Volkow N, Seamans J (2005) Unmanageable motivation in addiction: a pathology in prefrontal-accumbens glutamate transmission. Neuron 45:647-650.

Kasai H, Matsuzaki M, Noguchi J, Yasumatsu N, Nakahara H (2003) Structure-stability-function relationships of dendritic spines. Trends Neurosci 26:360-368.

Krause M, Dent EW, Bear JE, Loureiro JJ, Gertler FB (2003) Ena/VASP proteins: Regulators of the actin cytoskeleton and cell migration. Annu Rev Cell Dev Biol 19:541-564.

Lisman J (2003) Actin's actions in LTP-induced synapse growth. Neuron 38:361-362.

Lua BL, Low BC (2005) Cortactin phosphorylation as a switch for actin cytoskeletal network and cell dynamics control. FEBS Lett 579:577-585.

Majewska A, Sur M (2003) Motility of dendritic spines in visual cortex in vivo: changes during the critical period and effects of visual deprivation. Proc Natl Acad Sci USA 100:16024-16029.

Matsuoka Y, Li X, Bennett V (2000) Adducin: structure, function and regulation. Cell Mol Life Sci 57:884-895.

Matsuzaki M, Honkura N, Ellis-Davies GC, Kasai H (2004) Structural basis of long-term potentiation in single dendritic spines. Nature 429:761-766.

Matus A (2005) Growth of dendritic spines: a continuing story. Curr Opin Neurobiol 15:67-72.

May RC (2001) The Arp2/3 complex: a central regulator of the actin cytoskeleton. Cell Mol Life Sci 58:1607-1626.

McFarland K, Kalivas PW (2001) The circuitry mediating cocaine-induced reinstatement of drug-seeking behavior. J Neurosci 21:8655-8663.

McFarland K, Lapish CC, Kalivas PW (2003) Prefrontal glutamate release into the core of the nucleus accumbens mediates cocaine-induced reinstatement of drug-seeking behavior. J Neurosci 23:3531-3537.

McGee AW, Bredt DS (2003) Assembly and plasticity of the glutamatergic postsynaptic specialization. Curr Opin Neurobiol 13:111-118.

Mejillano MR, Kojima S-I, Applewhite DA, Gertler FB, Svitkina TM, Borisy GG (2004) Lamellipodial versus filopodial mode of the actin nanomachinery: pivotal role of the filament barbed end. Cell 118:363-373.

Meng Y, Zhang Y, Tregoubov V, Janus C, Cruz L, Jackson M, Lu WY, MacDonald JF, Wang JY, Falls DL, Jia Z (2002) Abnormal spine morphology and enhanced LTP in LIMK-1 knockout mice. Neuron 35:121-133.

Meng Y, Zhang Y, Tregoubov V, Falls DL, Jia Z (2003) Regulation of spine morphology and synaptic function by LIMK and the actin cytoskeleton. Rev Neurosci 14:233-240.

Morton WM, Ayscough KR, McLaughlin PJ (2000) Latrunculin alters the actin-monomer subunit interface to prevent polymerization. Nat Cell Biol 2:376-378. 
Nimchinsky EA, Sabatini BL, Svoboda K (2002) Structure and function of dendritic spines. Annu Rev Physiol 64:313-353.

Ono S (2003) Regulation of actin filament dynamics by actin depolymerizing factor/cofilin and actin-interacting protein 1: new blades for twisted filaments. Biochemistry 42:13363-13370.

Paxinos G, Watson C (1986) The rat brain in stereotaxic coordinates, Ed 2. New York: Academic.

Pierce RC, Bell K, Duffy P, Kalivas PW (1996) Repeated cocaine augments excitatory amino acid transmission in the nucleus accumbens only in rats having developed behavioral sensitization. J Neurosci 16:1550-1560.

Pollard TD, Borisy GG (2003) Cellular motility driven by assembly and disassembly of actin filaments. Cell 112:453-465.

Rabenstein RL, Addy NA, Caldarone BJ, Asaka Y, Gruenbaum LM, Peters LL, Gilligan DM, Fitzsimonds RM, Picciotto MR (2005) Impaired synaptic plasticity and learning in mice lacking $\beta$-adducin, an actin-regulating protein. J Neurosci 25:2138-2145.

Rao A, Craig AM (2000) Signaling between the actin cytoskeleton and the postsynaptic density of dendritic spines. Hippocampus 10:527-541.

Robinson TE, Kolb B (1997) Persistent structural modifications in nucleus accumbens and prefrontal cortex neurons produced by previous experience with amphetamine. J Neurosci 17:8491-8497.

Robinson TE, Kolb B (1999) Morphine alters the structure of neurons in the nucleus accumbens and neocortex of rats. Synapse 33:160-162.

Robinson TE, Gorny G, Mitton E, Kolb B (2001) Cocaine selfadministration alters the morphology of dendrites and dendritic spines in the nucleus accumbens and neocortex. Synapse 39:257-266.

Rosahl TW, Spillane D, Missler M, Herz J, Selig DK, Wolff JR, Hammer RE, Malenka RC, Sudhof TC (1995) Essential functions of synapsins I and II in synaptic vesicle regulation. Nature 375:488-493.

Soosairajah J, Maiti S, Wiggan O, Sarmiere P, Moussi N, Sarcevic B, Sampath R, Bamburg JR, Bernard O (2005) Interplay between components of a novel LIM kinase-slingshot phosphatase complex regulates cofilin. EMBO J 24:473-486.

Suto N, Tanabe LM, Austin JD, Creekmore E, Pham CT, Vezina P (2004) Previous exposure to psychostimulants enhances the reinstatement of cocaine seeking by nucleus accumbens AMPA. Neuropsychopharmacology 29:2149-2159.
Sutton MA, Schmidt EF, Choi KH, Schad CA, Whisler K, Simmons D, Karanian DA, Monteggia LM, Neve RL, Self DW (2003) Extinction-induced upregulation in AMPA receptors reduces cocaine-seeking behaviour. Nature 421:70-75.

Svitkina TM, Bulanova EA, Chaga OY, Vignjevic DM, Kojima S, Vasiliev JM, Borisy GG (2003) Mechanism of filopodia initiation by reorganization of a dendritic network. J Cell Biol 160:409-421.

Swanson C, Baker D, Carson D, Worley P, Kalivas P (2001) Repeated cocaine administration attenuates group I metabotropic glutamate receptor-mediated glutamate release and behavioral activation: a potential role for Homer 1b/c. J Neurosci 21:9043-9052.

Thomas MJ, Beurrier C, Bonci A, Malenka RC (2001) Long-term depression in the nucleus accumbens: a neural correlate of behavioral sensitization to cocaine. Nat Neurosci 4:1217-1223.

Toda S, Alguacil LF, Kalivas PW (2003) Repeated cocaine administration changes the function and subcellular distribution of adenosine Al receptor in the rat nucleus accumbens. J Neurochem 87:1478-1484.

Wang HG, Lu FM, Jin I, Udo H, Kandel ER, de Vente J, Walter U, Lohmann SM, Hawkins RD, Antonova I (2005) Presynaptic and postsynaptic roles of NO, cGK, and RhoA in long-lasting potentiation and aggregation of synaptic proteins. Neuron 45:389-403.

White FJ, Xiu Y-H, Henry DJ, Zhang X-F (1995) Neurophysiological alterations in the mesocorticolimbic dopamine system during repeated cocaine administration. In: The neurobiology of cocaine addiction (Hammer R, ed), pp 99-120. Boca Raton, FL: CRC.

Wolf ME (1998) The role of excitatory amino acids in behavioral sensitization to psychomotor stimulants. Prog Neurobiol 54:679-720.

Yang N, Higuchi O, Ohashi K, Nagata K, Wada A, Kangawa K, Nishida E, Mizuno K (1998) Cofilin phosphorylation by LIM-kinase 1 and its role in Rac-mediated actin reorganization. Nature 393:809-812.

Yao WD, Gainetdinov RR, Arbuckle MI, Sotnikova TD, Cyr M, Beaulieu JM, Torres GE, Grant SG, Caron MG (2004) Identification of PSD-95 as a regulator of dopamine-mediated synaptic and behavioral plasticity. Neuron 41:625-638.

Zhang X-F, Hu X-T, White FJ (1998) Whole-cell plasticity in cocaine withdrawal: reduced sodium current in nucleus accumbens neurons. J Neurosci 18:488-498. 